\title{
Flash-induced enhancements in the proton NMR relaxation rate of Photosystem II particles: response to flash trains of 1-5 flashes
}

\author{
A.N. Srinivasan and R.R. Sharp \\ Department of Chemistry, The University of Michigan, Ann Arbor, MI 48109 (U.S.A.)
}

(Received 21 April 1986)

Key words: Water splitting; NMR; Manganese; Photosystem II; Oxygen evolution; S-state transition

Flash-induced enhancements of the proton NMR relaxation rate $R_{1}$ of the solvent in suspensions of PS II particles have been recorded for sequences of 1-5 saturating flashes. The one-flash relaxation transient is a positive $R_{1}$ enhancement of $0.0080 \mathrm{~s}^{-1}$, which relaxes to the preflash baseline with a half-time of $25 \mathrm{~s}$. The two-flash response is a positive relaxation transient of nearly identical amplitude to the one-flash response, but with a slower decay $\left(t_{1 / 2} \approx 40 \mathrm{~s}\right)$. The appearance of a strongly relaxing paramagnetic center after one flash is consistent with the expected properties of an $\mathrm{Mn}(\mathrm{III}) \rightarrow \mathrm{Mn}$ (IV) oxidation. In contrast, the absence of a further $R_{1}$ enhancement after the second flash shows that a strongly relaxing paramagnetic center is not formed by $S_{2} \rightarrow S_{3}$ as is expected for manganese redox chemistry involving oxidation of Mn(III); the NMR experiment gives no indication that manganese redox chemistry occurs on the $S_{2} \rightarrow S_{3}$ transition. The three-flash $R_{1}$ response is a positive $R_{1}$ transient of $0.0066 \mathrm{~s}^{-1}$, which decays with a half-time of approx. 50 $s$ to a stable value of $0.002 \mathrm{~s}^{-1}$ above the preflash baseline. Positive $R_{1}$ enhancements have been observed in the presence of carbonylcyanide $m$-chlorophenylhydrazone (CCCP), an agent known to accelerate the decay of $S_{2}$ and $S_{3}$, at concentrations which very effectively suppress the one-flash and two-flash decays. These enhancements indicate that the formation of the $S_{0}$ state from $S_{1}$ involves the production of a strongly relaxing center, and the sign of the $R_{1}$ response is consistent with the expected properties of an $M n(I I I) \rightarrow M n(I I)$ reduction. The kinetic behavior of the three-flash $R_{1}$ transient is indicative of slow redox equilibria involving the strongly relaxing center of the $S_{0}$ state. Flash transients across a five-flash cycle exhibit oscillatory behavior with a local minimum on the fourth flash. Simulations of the flash profile indicate the presence of an $R_{1}$ contribution from a minor fraction of centers which are capable of transition to $S_{2}$ and $S_{3}$, but are inhibited at $S_{3} \rightarrow S_{0}$.

\section{Introduction}

The catalytic cycle of photosynthetic water oxidation is known to involve a manganese cluster

Abbreviations: Mes, 4-morpholineethanesulfonic acid; Chl, chlorophyll; PS, Photosystem; CCCP, carbonylcyanide mchlorophenylhydrazone; ADRY, acceleration of the deactivation reactions of the water-oxidizing enzyme system; DCBQ, 3,5-dichlorobenzoquinone.

Correspondence address: Dr. R.R. Sharp, Department of Chemistry, The University of Michigan, Ann Arbor, MI 48109, U.S.A. which is driven by the photochemistry of PS II through a sequence of five oxidation states, the so-called $\mathrm{S}$ states $\left(\mathrm{S}_{0}, \mathrm{~S}_{1}, \mathrm{~S}_{2}, \mathrm{~S}_{3}, \mathrm{~S}_{4}\right)$. These oxidation states of the manganese center mediate the four-electron oxidation of water to molecular oxygen $[1,2]$. Absolute manganese requirement for the functioning of the water oxidation is well documented. Quantitation of manganese in PS II has indicated a stoichiometry of four tightly bound manganese ions per center [3-6], although a value of 2 [7] has also been reported.

Recently, spectroscopic probes of the S states 
have been developed using ESR [8-10] and optical [11-16] spectroscopy. ESR signals apparently arise from two different forms of the $S_{2}$ state, one a multiline signal centered about $g=2$ and the other a broad absorption at $g=4.1$. These data suggest that the $S_{2}$ state consists of a mixed valence manganese cluster. An optical transient in the near infrared supports this interpretation [16]. Period-4 optical transients have also been observed in flash studies in the ultraviolet [12-14]. The analysis of these experiments has yielded four optical transients during the four S-state transitions. These transients, which are similar in nature, have been interpreted in terms of four sequential $\mathrm{Mn}(\mathrm{III}) \rightarrow \mathrm{Mn}(\mathrm{IV})$ oxidations during the S-state cycle [14].

Recent work from this laboratory [17] has shown that the $S_{1} \rightarrow S_{2}$ transition produces a detectable enhancement of the NMR $R_{1}$ relaxation rate of the solvent protons in suspensions of PS II particles. This NMR relaxation enhancement monitors the appearance of a strongly relaxing paramagnetic center which is produced during the $S_{1} \rightarrow S_{2}$ transition. The sign of the proton NMR relaxation enhancement is consistent with the expected properties of a $\mathrm{Mn}(\mathrm{III}) \rightarrow \mathrm{Mn}(\mathrm{IV})$ oxidation. Evidence linking the NMR relaxation transient to redox chemistry in the $\mathrm{S}$ states derives from a variety of observations. These include: (1) the decay kinetics of the transient, which correspond to known properties of S-state decay; (2) the suppression of the NMR transient by treatments which release manganese from PS II (washing by alkaline Tris buffer or $1 \mathrm{mM} \mathrm{NH}{ }_{2} \mathrm{OH}$ ); (3) the sensitivity of the response to $\mathrm{CCCP}$, an agent which accelerates S-state decay; (4) the observation that subextracting concentrations of $\mathrm{NH}_{2} \mathrm{OH}$, which are known to produce a two-flash retardation in the normal period-4 cycle of oxygen flash yields, also produce a two-flash delay in the appearance of the NMR relaxation transient; and (5) the ineffectiveness of atrazine-type inhibition of electron transport on the reducing side of PS II in suppressing the one-flash $R_{1}$ response.

In this communication we present a characterisation of the proton NMR relaxation enhancement transient in suspensions of PS II particles across a five-flash cycle. The purpose of these measurements is the development of an experimental probe of manganese redox chemistry that occurs during the $S_{2} \rightarrow S_{3}$ and $S_{0} \rightarrow S_{1}$ transitions.

\section{Materials and Methods}

PS-II-enriched membranes were prepared from market spinach by the procedure of Berthold et al. [18] as modified by Ghanotakis et al. [19]. The PS II membranes were suspended in a buffer containing $400 \mathrm{mM}$ sucrose $/ 15 \mathrm{mM} \mathrm{NaCl} / 40 \mathrm{mM} \mathrm{Na}$ Mes at $\mathrm{pH} 6.0$, divided into $0.75 \mathrm{ml}$ aliquots and stored at $190 \mathrm{~K}$ until use. Typical control rates for oxygen evolution activity were $400-450 \mu \mathrm{mol} \mathrm{O}_{2}$ per mg Chl per h.

Samples were dark adapted for a minimum of 3 $h$ on ice prior to start of measurements. Samples were kept in total darkness except when flash-illuminated. Addition of $0.5 \mathrm{mM}$ EDTA, electron acceptor and other reagents (as detailed in figure legends) was made after the dark adaptation period.

NMR samples consisted of $30 \mu 1$ of the PS II suspension at a concentration equivalent to $1.9 \mathrm{mg}$ $\mathrm{Chl}$ per $\mathrm{ml}$. The flash measurements utilized a cylindrical flat cell $(8 \mathrm{~mm}$ diameter by $0.5 \mathrm{~mm}$ depth) milled into a lucite light pipe as described in Refs: 17 and 20. Samples were temperatureequilibrated in the NMR probe at $18-20^{\circ} \mathrm{C}$ for 10 min and then illuminated with a single actinic flash 6-6.5 min before beginning the measurement sequence. Details of the NMR instrumentation and the basic measurement sequence are described in Refs. 17 and 21

Flash illumination was provided by a Xenon Corporation model 457 power supply and a Novatron-722 flash lamp. Actinic flashes had a half-width of about $10 \mu \mathrm{s}$ and were generated by dissipating electrical energy of $70 \mathrm{~J}$ per pulse. The flash output was collected by a parabolic mirror and focused into the sample chamber by means of a condensing lens, a right-angle prism, and a light pipe [17]. A saturation curve of the one flash proton NMR relaxation enhancement transient was recorded as a function of the voltage across the discharge capacitor. Flash energies higher than $50 \mathrm{~J}$ per pulse were found to be saturating. 


\section{Results}

Enhancements of the NMR relaxation rates $\left(R_{1}\right)$ of solvent water protons produced by actinic flash trains on PS II suspensions are shown in Figs. 1-3. In all experiments, samples were dark adapted for $3 \mathrm{~h}$ at $0^{\circ} \mathrm{C}$ and then illuminated with a single flash 6-6.5 min before commencement of the measurement sequence. This procedure maximizes the fraction of manganese centers in the $\mathrm{S}_{1}$-state at the start of experiments.

Figs. 1a and 2a show the proton NMR relaxation enhancement transients produced by one flash and two flashes, repsectively. Amplitudes of the transients measured $150 \mathrm{~ms}$ after the final flash are 0.0080 and $0.0079 \mathrm{~s}^{-1}$. These transients decay to the baseline with half-times of about $25 \mathrm{~s}$ (one flash) and $40 \mathrm{~s}$ (two flashes).

Fig. $1 \mathrm{~b}$ and $2 \mathrm{~b}$ show the effect of $1 \mu \mathrm{M} \mathrm{CCCP}$ on the one-flash and two-flash $R_{1}$ transients. CCCP is known to accelerate the deactivation of the higher $S$ states $\left(S_{2}\right.$ and $\left.S_{3}\right)$ back to the $S_{1}$ state

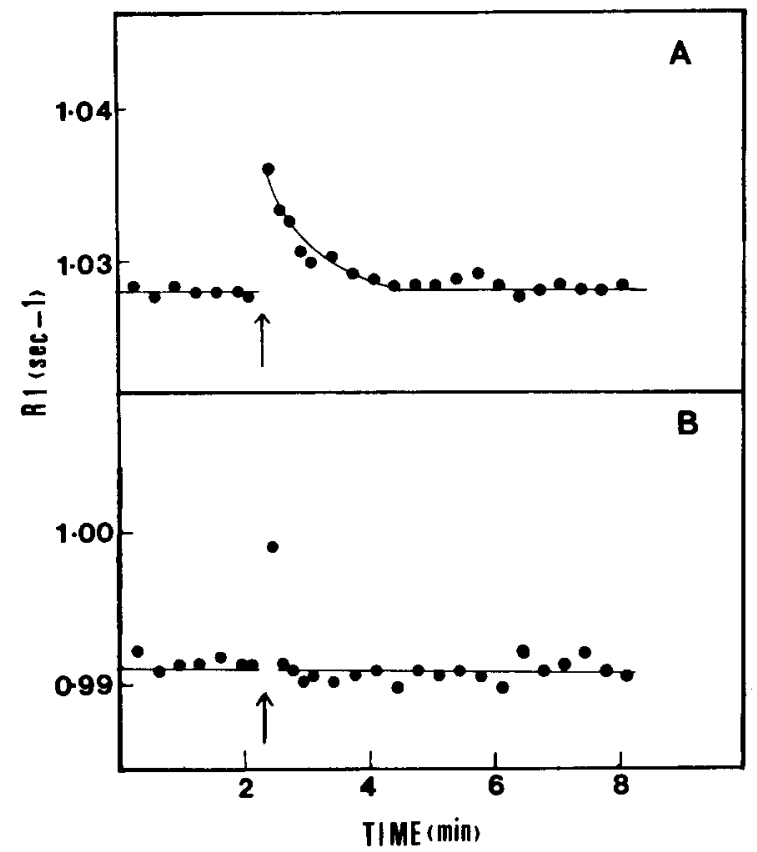

Fig. 1. Proton-solvent relaxation rate as a function of time in a suspension of PS II particles following a single actinic flash. Samples contained $1.5 \mathrm{mM} \mathrm{Fe}(\mathrm{CN})_{6}^{-3}$ and $0.5 \mathrm{mM}$ EDTA. (A) was without ADRY reagent, (B) contained $1 \mu \mathrm{M}$ CCCP. Chlorophyll concentration was $1.87 \mathrm{mg} \cdot \mathrm{ml}^{-1}$. Each trace is an average of 25 experiments.
(ADRY effect). This effect is clearly evident in both the one-flash and two-flash $R_{1}$ transients. A CCCP titration [17] indicates that the $R_{1}$ response at $10 \mathrm{~s}$ is effectively suppressed by $\mathrm{CCCP}$ concentrations of at least $0.5 \mu \mathrm{M}$. In the presence of 1 $\mu \mathrm{M}$ CCCP, only the first point of each sequence, measured $150 \mathrm{~ms}$ after the flash, is unaffected. While the one flash response decays abruptly to the baseline, in the two-flash sequence a small, but observable, decay persists $10 \mathrm{~s}$ after the final flash. This is probably due to effects of double hits, which produce a small fraction of centers in the $S_{0}$ state.

Fig. 3 shows the $R_{1}$ response following three flashes in the absence (Fig. 3a) and presence (Fig. $3 b)$ of ADRY reagent. Three flashes are expected to produce a maximum number of centers in the $\mathrm{S}_{0}$ state, which in thylakoid membranes is relatively stable over periods of several tens of minutes. The observed proton NMR relaxation enhancement decays, following the third flash, from an initial amplitude of $0.0074 \mathrm{~s}^{-1}$ to a final, relatively stable enhancement $0.0020 \mathrm{~s}^{-1}$ above the preflash baseline. In order to distinguish the true, stable

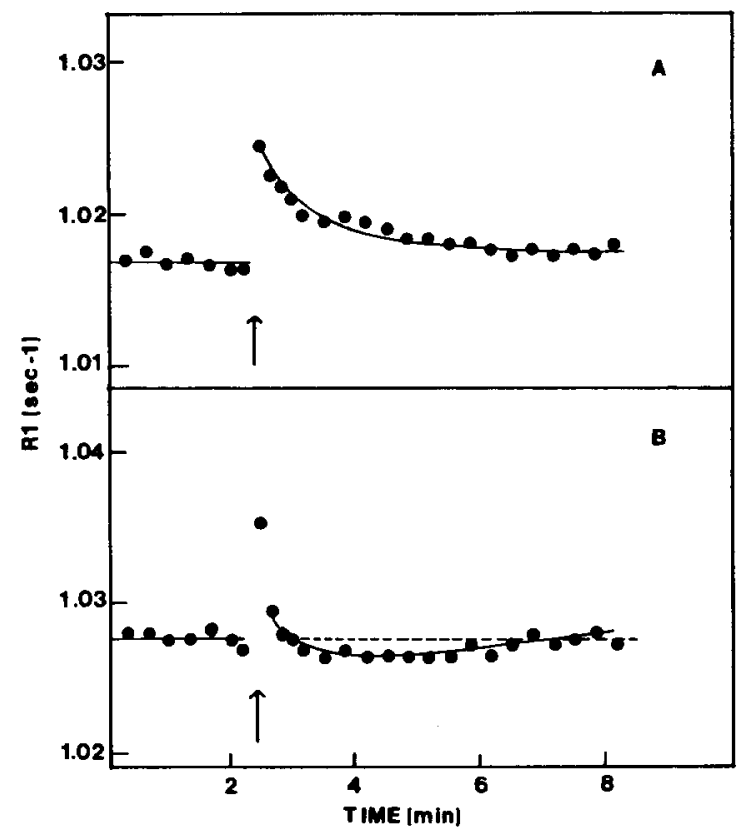

Fig. 2. Proton-solvent relaxation rate as a function of time in a suspension of PS II particles following two actinic flashes spaced $300 \mathrm{~ms}$ apart. Other experimental conditions are given in the legend of Fig. 1. 


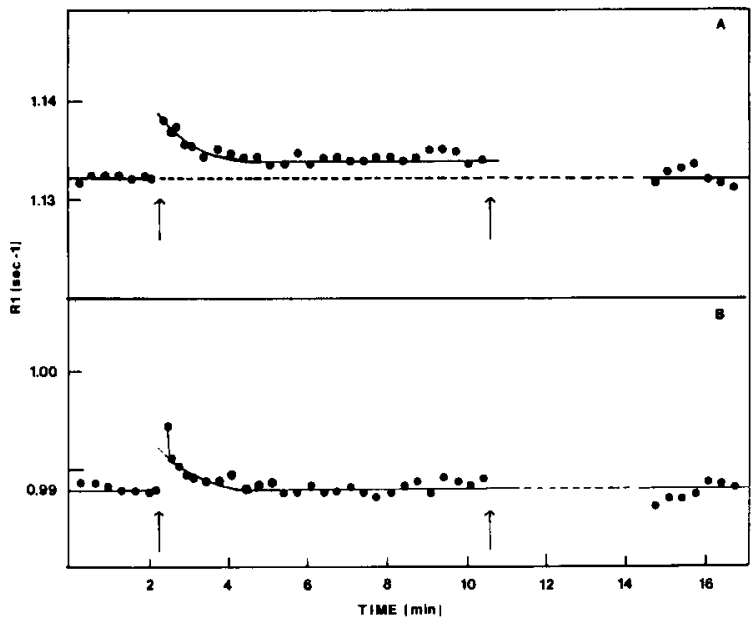

Fig. 3. Proton-solvent relaxation rate as a function of time in a suspension of PS II particles following three actinic flashes spaced $300 \mathrm{~ms}$ apart. $8 \mathrm{~min}$ after the flash train, a single flash was applied followed by a $3.5 \mathrm{~min}$ dark delay to reset the $S$-state system to the $S_{1}$ state. Samples contained $1.5 \mathrm{mM}$ $\mathrm{Fe}(\mathrm{CN})_{6}^{-3}$ and $0.5 \mathrm{mM}$ EDTA with (B) or without (A) $1 \mu \mathrm{M}$ CCCP. Chlorophyll concentration is $1.87 \mathrm{mg} \cdot \mathrm{ml}^{-1}$. Each trace is an average of 24 experiments.

enhancement from effects of slow $R_{1}$ variation due to temperature drift, a single flash, followed by a $3.5 \mathrm{~min}$ dark delay, was applied $8 \mathrm{~min}$ after the three-flash sequence (indicated by the second arrow in Fig. 3). This second flash returns all centers to the $S_{1}$ state and thus defines the postsequence baseline for the three-flash experiment. The $S_{1}$ baseline is shown by dashed lines in Figs. $3 a$ and $b$.

The three-flash sequence produces a positive $R_{1}$ transient, which decays with a half-time of approx. $40 \mathrm{~s}$ to a stable $\Delta R_{1}$ value of $0.002 \mathrm{~s}^{-1}$. The decaying component of the transient was unexpected in terms of the presumed stability of $S_{0}$ state; it was initially interpreted as resulting from centers in the $S_{2}$ and $S_{3}$ states, due either to the use of non-saturating actinic flashes, or due to damaged centers unable to complete the $S_{3} \rightarrow S_{4}$ $\rightarrow S_{0}$ transition. However, the addition of CCCP at a concentration sufficient to suppress entirely the $S_{2}$ and $S_{3}$ responses other than the first measured point, did not similarly suppress the three flash response. In the presence of CCCP, the $R_{1}$ measurements collected at times greater than $10 \mathrm{~s}$ after the three flash sequence reflect exclusively centers taken to the $S_{0}$ state. Thus the response

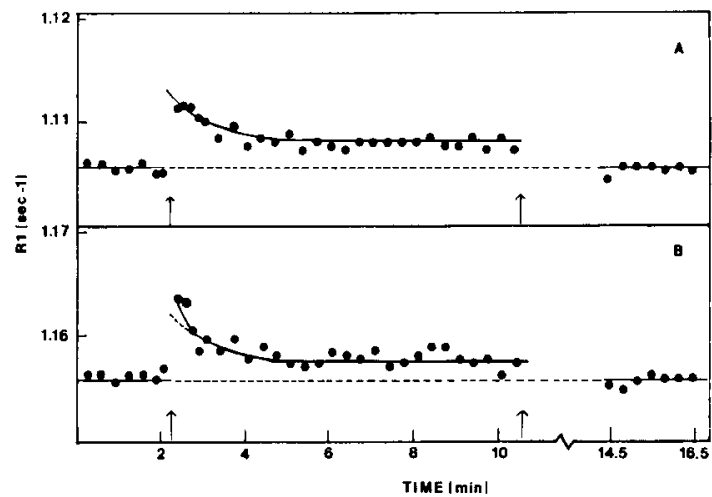

Fig. 4. Proton-solvent relaxation rate as a function of time in a suspension of PS II particles following three actinic flashes. Experimental conditions are the same as described in Fig. 3 except for the inclusion of $250 \mu \mathrm{M} \mathrm{DCBQ}$ in the suspension in place of $\mathrm{Fe}(\mathrm{CN})_{6}^{-3}$.

due to $\mathrm{S}_{1} \rightarrow \mathrm{S}_{0}$ is a positive $R_{1}$ transient, which exhibits both kinetic and stationary components. The kinetic component, for which $\tau_{1 / 2} \approx 50 \mathrm{~s}$, was observed both in the absence and presence of CCCP. In contrast, the stationary component, which reflects relaxation due to a stable para-

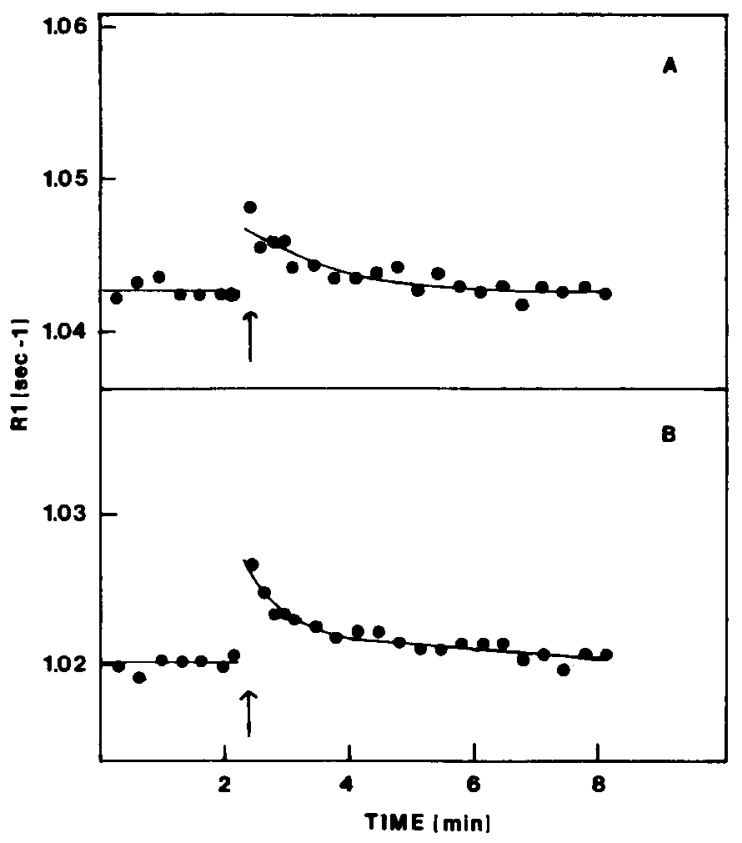

Fig. 5. Proton-solvent relaxation rate as a function of time in a suspension of PS II particles following four (A) and five (B) actinic flashes spaced $300 \mathrm{~ms}$ apart. Other experimental conditions are given in the legend of Fig. 1 . 
magnetic center in the $S_{0}$ state, was suppressed by CCCP.

This behavior suggested that the strongly relaxing center in $\mathrm{S}_{0}$ might be oxidizable by $\mathrm{Fe}\left(\mathrm{CN}_{6}\right)^{-3}$ when $\mathrm{CCCP}$ is present as a redox mediator. To test this hypothesis, the 3-flash experiment was repeated using a weaker oxidant, DCBQ, as electron acceptor. Three flashes again produced a stable relaxation enhancement (Fig. 4A), which in this case persisted in the presence of CCCP (Fig. 4B). Thus the data of Figs. 3 and 4 demonstrate the presence of a strongly relaxing center in the $S_{0}$ state and suggest that this center may be oxidizable by $\mathrm{Fe}\left(\mathrm{CN}_{6}\right)^{-3}$, but not $\mathrm{DCBQ}$, in the presence of CCCP.

Fig. 5 shows the proton NMR relaxation enhancement transient following the trains of four (Fig. 5a) and five (Fig. 5b) flashes. A positive, decaying $R_{1}$ enhancement is seen in both experiments. Fig. 6 contains a plot of the initial amplitude of the proton NMR relaxation enhancement transients as a function of flash number across the five flash cycle. In this cycle, the fourth flash represents a nominal return of the manganese center to the $S_{1}$ state and is expected to produce a local minimum in the flash response. This expectation is realized, although the amplitude of the oscillation is less than that normally encountered in measurements of $\mathrm{O}_{2}$ flash yields. Effects leading to damping of the $R_{1}$ oscillation are considered further below.

\section{Discussion}

The efficiency with which membrane-bound paramagnetic ions are able to act as relaxation traps for solvent protons depends strongly on the oxidation state of the ion. Specifically, strongly relaxing centers are those which possess large paramagnetic moments and for which the electronic spin relaxation time is long, i.e., comparable to the inverse proton Larmor frequency (approx. $10^{-8} \mathrm{~s}$ at $20 \mathrm{MHz}$ ). Such long paramagnetic relaxation times are characteristic of ions in orbital singlet states, where the static zero-field splitting is small [22]. Among the various common manganese oxidation states, only $\mathrm{Mn}$ (II) and $\mathrm{Mn}(\mathrm{IV})\left({ }^{6} \mathrm{~A}_{1}\right.$ and ${ }^{4} \mathrm{~A}_{2}$, respectively, in octahedral environments) possess orbital singlet electronic

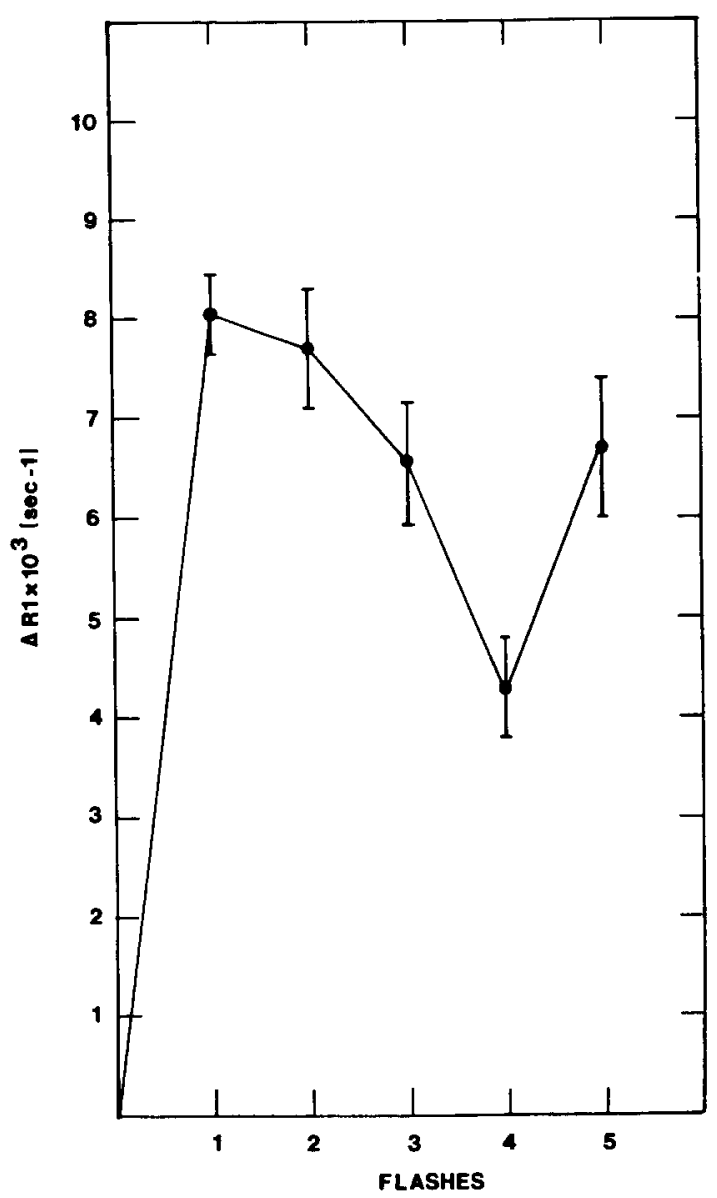

Fig. 6. Amplitude of the flash induced $R_{1}$ transient in suspensions of PS II particles as a function of flash number.

ground states. Thus the proton NMR relaxation enhancement experiment provides a reasonably selective probe of the appearance of disappearance of these two oxidation states during Sstate transitions.

Fig. 6 summarizes the amplitudes of the observed proton NMR relaxation enhancement transients across a 5-flash cycle. In interpreting these transients in terms of the probable underlying manganese redox chemistry, we take the appearance of a strongly relaxing center on an oxidative S-state transition as evidence for a $\mathrm{Mn}(\mathrm{III}) \rightarrow \mathrm{Mn}(\mathrm{IV})$ oxidation; the appearance of a strongly relaxing center on a reductive S-state transition is evidence for a $\mathrm{Mn}$ (III) $\rightarrow \mathrm{Mn}$ (II) reduction. We believe that an interpretation based on individual ion oxidation states is probably ap- 
propriate for the S-state system at room temperature. However, we cannot at present exclude the possible involvement of very strongly exchangecoupled manganese dimers or tetramers, in which case the observation of efficient NMR relaxation traps would be associated chemically with strongly spin-coupled spin- $\frac{1}{2}$ centers.

The $S_{1} \rightarrow S_{2}$ transition is accompanied by the appearance of a strongly relaxing center, clearly indicative of a $\mathrm{Mn}$ (III) $\rightarrow \mathrm{Mn}$ (IV) oxidation. The behavior of this transient has been described in detail previously [17]. The two-flash response is essentially identical in amplitude to the one-flash response although slower in its decay. Thus the second flash does not produce a second strongly relaxing center; the proton NMR relaxation enhancement experiment gives no indication that manganese redox chemistry takes place on this transition. The slowed decay after two flashes evidently reflects the altered kinetics of charge recombination between the $S$ states and the quinone $Q_{B} / Q_{B}^{-}$on the acceptor side of PS II. Assuming that the acceptor side is largely oxidized during the dark-adaptation period, a single flash produces $\mathrm{Q}_{\mathrm{B}}^{-}$, and two flashes produce $\mathrm{Q}_{\mathrm{B}}$. Recombination from $Q_{B}^{-}$to the $S$ states is a more efficient process than is recombination from $Q_{B}$.

The relaxation properties of the $S_{1} \rightarrow S_{0}$ transition are described most clearly by the three flash experiments of Figs. 3 and 4. In summary, the $S_{1} \rightarrow S_{0}$ transition produces a strongly relaxing center which exhibits both kinetic and stationary components. In the absence of ADRY reagent, three flashes produce a positive $R_{1}$ enhancement which persists over the time-course of the experiment. In the presence of ADRY reagent (CCCP, 1 $\mu \mathrm{M}$ ) however, the three-flash $R_{1}$ enhancement relaxes to the $S_{1}$ baseline when the redox poise of the medium is strongly oxidizing $(1.5 \mathrm{mM}$ $\left.\mathrm{Fe}(\mathrm{CN})_{6}^{-3}\right)$. Under less strongly oxidizing conditions (250 $\mu \mathrm{M}$ DCBQ), a stable $R_{1}$ enhancement persists both in the presence and absence of ADRY.

These results indicate that a strongly relaxing center is formed during the $S_{1} \rightarrow S_{0}$ transition. The appearance of a strongly relaxing center on a reductive transition of the $S$ states is suggestive of the formation of $\mathrm{Mn}$ (II) from $\mathrm{Mn}$ (III). The $\mathrm{S}_{0}$ state can be oxidized by $\mathrm{Fe}(\mathrm{CN})_{6}^{-3}$ in the dark to a state that is identical in its NMR relaxation properties to $S_{1}$. This oxidation reaction is accelerated by the ADRY reagent CCCP. When the redox posie is set by $D C B Q$, the strongly relaxing center associated with the $S_{0}$ state is stable both in the presence and absence of CCCP, evidently due to the lower midpoint potential of this acceptor system. It is interesting to note, however, that the $R_{1}$ response measured in the presence of DCBQ and CCCP (Fig. 4b) contains both kinetic and stationary components. The kinetic component, for which $t_{1 / 2} \approx 50 \mathrm{~s}$, evidently reflects a partial CCCP-catalysed oxidation of the $S_{0}$ state.

The conclusion from the NMR data that the $\mathrm{S}_{1} \rightarrow \mathrm{S}_{2}$ transition involves a $\mathrm{Mn}(\mathrm{III}) \rightarrow \mathrm{Mn}(\mathrm{IV})$ oxidation is in accord with other experimental observations. In particular, the formation at low temperature of a multiline EPR signal on this transition indicates that manganese redox chemistry is involved, as does the observed shift to higher energy of the mangnese $K_{\alpha}$ absorption edge. The $S_{2}$ state evidently comprises a mixed valence manganese cluster $[8,16,23]$.

On the other hand, the apparent absence of manganese oxidation on the $S_{2} \rightarrow S_{3}$ transition in the proton NMR relaxation enhancement experiment conflicts with the conclusion of Dekker et al. [14], who attributed a broad optical transient near $315 \mathrm{~nm}$ to a $\mathrm{Mn}(\mathrm{III}) \rightarrow \mathrm{Mn}(\mathrm{IV})$ oxidation. However, other experimental observations appear consistent with the absence of manganese redox chemistry on this transition. In particular, the manganese $K_{\alpha}$ absorption edge is relatively little perturbed by the $S_{1} \rightarrow S_{3}$ transition, in contrast to its behavior after one flash [24]. Moreover, the thermoluminescence band associated with the $\mathrm{S}_{2} \cdot \mathrm{Q}_{\mathrm{B}}^{-}$recombination occurs at essentially the same temperature as that due to $S_{3} \cdot Q_{B}^{-}$recombination ( $B$ band) [25-30], suggesting that the holes associated with $S_{2}$ and $S_{3}$ may have similar potentials.

If the $S_{2} \rightarrow S_{3}$ transition involves oxidation of $\mathrm{Mn}(\mathrm{III})$ as inferred from the ultraviolet transients, then the Mn(IV) formed does not provide an efficient relaxation trap for proton magnetisation. This situation could occur if the Mn(IV) produced were magnetically isolated from the pool of solvent protons. Mechanistic studies of the relaxation coupling in the proton NMR relaxation enhancement 
experiment would be useful in this regard to assess whether magnetic coupling from manganese to the solvent protons involves a spin-diffusion mechanism [17]. If spin-diffusion coupling through the membrane dominates the relaxation pathway, magnetic isolation of manganese would be unlikely.

Our conclusion, based on the proton NMR relaxation enhancement data, that the $S_{0} \rightarrow S_{1}$ transition is associated with a $\mathrm{Mn}(\mathrm{II}) \rightarrow \mathrm{Mn}(\mathrm{III})$ oxidation also conflicts with the work of Dekker

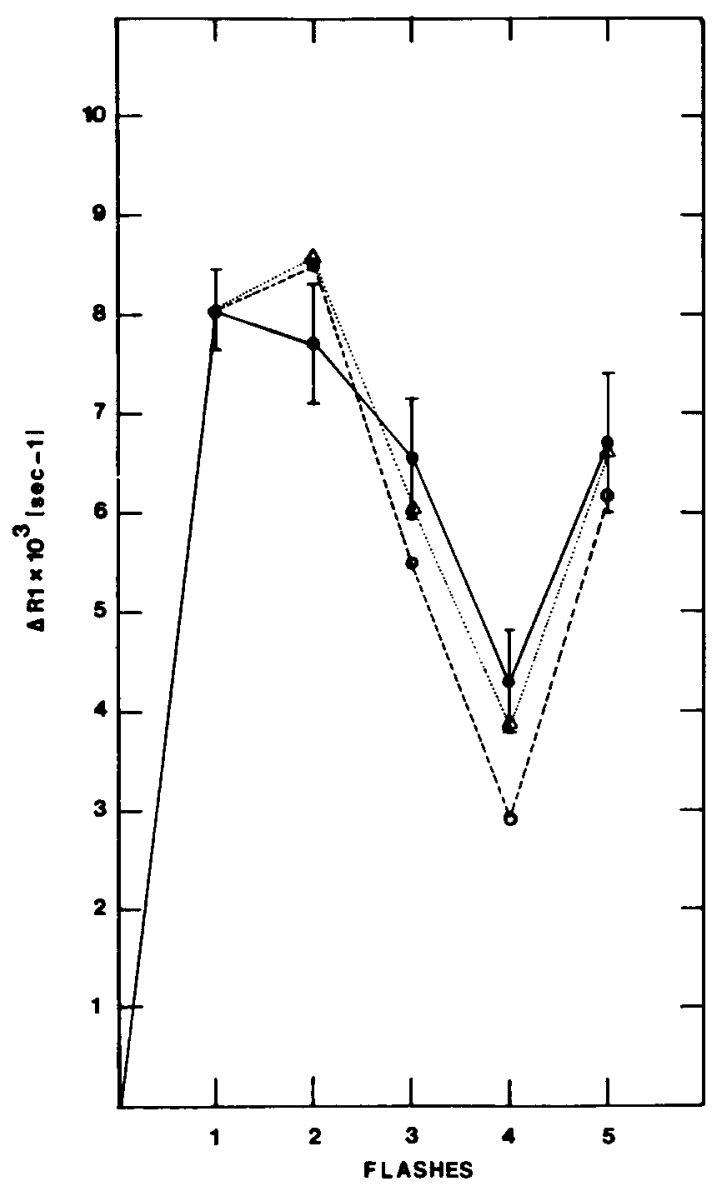

Fig. 7. Comparison of experimental and calculated proton NMR relaxation enhancement flash profiles. Solid line represents experimental points. $(\ldots .$.$) is a simulation assuming a$ misses parameter $\alpha=0.1$ and a double hit parameter $\beta=0.05$ $(\cdots \cdots)$ is a simulation assuming the same misses and double hit parameters, and also assumes a 0.15 fraction of damaged centers which are unable to cycle past the $S_{3}$ state. Both simulations are normalized to the experimental transient on the first flash. et al. [14], who interpreted the $315 \mathrm{~nm}$ optical transient in terms of $\mathrm{Mn}(\mathrm{III}) \rightarrow \mathrm{Mn}(\mathrm{IV})$. The chemical identity of the strongly relaxing center associated with $S_{0}$ can be further characterized through a study of the magnetic field dependence of the proton NMR relaxation enhancement. Such studies should clarify the proposed involvement of Mn(IV).

The proton NMR relaxation enhancement transients across a five-flash cycle are compared to theoretical simulations in Fig. 7. The overall S-state transient following the $j$ th flash is given by the expression

$T_{i}=\sum_{i=0}^{3} P_{i} T_{i}$

where $P_{i}$ is the fractional population of the $\mathrm{S}_{i}$ state and $T_{i}$ is the $R_{1}$ transient for the $\mathrm{S}_{1} \rightarrow \mathrm{S}_{i}$ transition assuming $100 \%$ efficiency. S-state populations were calculated assuming a misses parameter of $\alpha=0.1$ and a double-hits parameter of $\beta=0.05$. The proton NMR relaxation enhancement experiment, unlike $\mathrm{O}_{2}$ flash yields, is also sensitive to centers which are capable of oxidation to $S_{2}$ or $S_{3}$, but are incapable of completing the $\mathrm{S}$-state cycle. For this reason, simulations were based on two different assumptions with respect to the presence of inactive centers. The first (- . . . - ) assumed that all centers were active in $\mathrm{O}_{2}$ evolution, while the second $(\cdots \cdots)$ assumed that $15 \%$ of the centers were incapable of S-state turnover beyond $S_{3}$. The amplitudes of the flash-induced $R_{1}$ transients were taken from the data of Figs. 1a, 2a, and 3b. $T_{1}$ is by definition zero. $T_{3}$ was taken equal to $T_{2}$, which is the $\Delta R_{1}$ value following a single flash, normalized to $100 \%$ $\mathrm{S}_{2}+\mathrm{S}_{3}$ population. $T_{0}$ was the $\Delta R_{1}$ value measured $10 \mathrm{~s}$ after the 3 -flash sequence in the presence of CCCP, also normalized to $100 \% \mathrm{~S}_{0}$ population. $R_{1}$ data accumulated $10 \mathrm{~s}$ after the third flash were used to calculate $T_{0}$, since they contained contributions due only to $S_{0}$. Both simulations are normalized to the experimental oneflash transient.

The simulation which assumes that all centers are able to complete the S-state cycle was in only fair agreement with experiment on the fourth flash transient, where the calculated enhancement was 
substantially lower than the observed value. Inclusion of a small fraction of partially inactive centers improved the agreement at the third, fourth and fifth flashes. Measurements of the $R_{1}$ flash response were undertaken using several different PS II preparations, and the results shown in Fig. 6 were typical, i.e., the four-flash response was significantly greater than predicted assuming the absence of blocked centers. It is possible that partially blocked centers may result from loss of the $33 \mathrm{kDa}$ peripheral polypeptide, which is known to be required for the $S_{3} \rightarrow S_{0}$ transition.

\section{Acknowledgement}

Partial support of this research was obtained in the form of a grant from the United States Department of Agriculture Competitive Grants Research Program (No. 82-CRCR-1-1047).

\section{References}

1 Kok, B., Forbush, B. and McGloin, M. (1970) Photochem. Photobiol. 11, 457-475

2 Forbush, B., Kok, B. and McGloin, M. (1971) Photochem. Photobiol. 14, 307-321

3 Cheniae, G.M. and Martin, I.F. (1970) Biochim. Biophys. Acta 197, 219-239

4 Cheniae, G.M. and Martin, I.F. (1971) Plant Physiol. 47, 568-575

5 Radmer, R. and Cheniae, G.M. (1977) Topics in Photosynthesis 2, 303-348

6 Yocum, C.F., Yerkes, C.T., Blankenship, R.E., Sharp, R.R. and Babcock, G.T. (1981) Proc. Natl. Acad. Sci. USA 78, $7507-7511$

7 Yamamoto, Y. and Nishimura, M. (1983) Biochim. Biophys. Acta 724, 294-297

8 Dismukes, G.C., Ferris, K. and Watnick, P. (1982) Photobiochem. Photobiophys. 3, 243-256

9 Brudvig, G.L., Casey, J.L. and Sauer, K. (1983) Biochim. Biophys. Acta 723, 366-371

10 Zimmerman, J.L. and Rutherford, A.W. (1984) Biochim. Biophys. Acta 767, 160-167
11 Velthuys, B.R. (1981) in Proceedings of the 5th International Congress on Photosynthesis (Akoynoglou, G., ed.), Vol. 2, pp. 75-85, Balaban International Science Services, Philadelphia, PA

12 Renger, G. and Weiss, W. (1983) Biochim. Biophys. Acta $722,1-11$

13 Dekker, J.P., Van Gorkom, H.J., Brok, M. and Ouwehand, L. (1984) Biochim. Biophys. Acta 764, 301-309

14 Dekker, J.P., Van Gorkom, H.J., Wensink, J. and Ouwehand, L. (1984) Biochim. Biophys. Acta 767, 1-9

15 Dekker, J.P., Plijter, J.J., Ouwehand, L. and Van Gorkom, H.J. (1984) Biochim. Biophys. Acta 767, 176-179

16 Dismukes, G.C. and Mathis, P. (1984) FEBS Lett. 178, 51-54

17 Srinivasan, A.N. and Sharp, R.R. (1986) Biochim. Biophys. Acta 850, 211-217

18 Berthold, D.A., Babcock, G.T. and Yocum, C.F. (1981) FEBS Lett. 134, 231-234

19 Ghanotakis, D.F., Babcock, G.T. and Yocum, C.F. (1984) FEBS Lett. 167, 127-130

20 Sharp, R.R. and Yocum, C.F. (1981) Biochim. Biophys Acta $635,90-104$

21 Haddy, A.E., Frasch, W.D. and Sharp, R.R. (1985) Biochemistry $24,7926-7930$

22 Lewis, W.B. and Morgan, L.O. (1968) Paramagnetic Relaxatioin in Solutions in: Transition Metal Chemistry (Carlin, R.L., ed.), Vol. 4, pp. 33-112, Marcel Dekker, New York

23 Dismukes, G.C. and Siderer, Y. (1981) Proc. natl. Acad. Sci. USA 78, 274-298

24 Goodin, D.B., Yachandra, V.K., Britt, R.D., Sauer, K. and Klein, M.P. (1984) Biochim. Biophys. Acta 767, 209-216

25 Demeter, S. and Vass, I. (1984) Biochim. Biophys. Acta $764,24-32$

26 Rutherford, A.W. and Inoue, Y. (1984) Biochim. Biophys. Acta $764,24-32$

27 Ono, T. and Inoue, Y. (1985) Biochim. Biophys. Acta 806 331-340

28 Inoue, Y. (1983) in The Oxygen Evolving System of Photosynthesis (Inoue, Y., Murata, N., Crofts, A.R., Renger, G., Govindjee, and Satoh, K., eds.), Academic Press, New York

29 Rutherford, A.W., Renger, G., Koike, H. and Inoue, Y (1984) Biochim. Biophys. Acta 767, 548-556

30 Rutherford, A.W., Crofts, A.R. and Inoue, Y. (1982) Biochim. Biophys. Acta 682, 457-465 